\title{
No open or flat bouncing cosmologies in Einstein gravity
}

\author{
Maulik Parikh \\ Department of Physics and Beyond: Center for Fundamental Concepts in Science, \\ Arizona State University, \\ Tempe, AZ 85287, U.S.A. \\ E-mail: maulik.parikh@asu.edu
}

\begin{abstract}
We show that, to first approximation, strings cannot propagate in bouncing open or flat Friedmann-Robertson-Walker universes. Specifically, the Virasoro constraint translates to the Ricci convergence condition in spacetime at leading order in the alphaprime expansion. Thus one must go beyond minimally-coupled classical Einstein gravity in order to find bounce solutions that could be consistent with string theory. We map out some remaining possibilities for finding string-compatible cosmological bounces.
\end{abstract}

Keywords: Classical Theories of Gravity, Bosonic Strings

ARXIV EPRINT: 1501.04606 


\section{Contents}

1 Introduction 1

2 Derivation of the Ricci convergence condition 3

$\begin{array}{llr}3 & \text { Implications for bouncing cosmologies } & 6\end{array}$

\section{Introduction}

To evade the Big Bang singularity, cosmologists have often envisioned the existence of a cosmological bounce, whereby the scale factor of the universe has a nonzero minimum, with a contracting phase preceding the present expansionary era. This idea, which dates back at least to the "Tolman wormhole" [1], has seen many contemporary reincarnations (see, e.g., $[2,3]$ and references therein).

However, to realize this scenario within the context of a classical flat or open FriedmannRobertson-Walker universe, it is well known that Einstein's equations require that the null energy condition be violated. ${ }^{1}$ Consider an FRW universe in $d+1$ spacetime dimensions sourced by a fluid with energy density $\rho$ and pressure $p$, and with cosmological constant $\Lambda$. Einstein's equations are

$$
\begin{aligned}
H^{2} & =\frac{16 \pi G_{N}}{d(d-1)} \rho-\frac{k}{a^{2}}+\frac{2 \Lambda}{d(d-1)} \\
\dot{H} & =-\frac{8 \pi G_{N}}{d-1}(\rho+p)+\frac{k}{a^{2}} .
\end{aligned}
$$

Since $\dot{H}=\frac{\ddot{a}}{a}-H^{2}$, and $H$ vanishes at the bounce, the left-hand side of (1.2) is equal to $\frac{\ddot{a}}{a}$ at the moment of the bounce. Thus, for $k \neq 1$, positivity of $\ddot{a}$, which is a sufficient condition for a bounce, requires that $\rho+p<0$, in violation of the NEC. This too-quick argument has a tiny loophole: the strict positivity of $\ddot{a}$ at $t=0$ (the moment of the bounce) is a sufficient but not a necessary condition for a bounce to exist. For example, a scale factor that near the bounce was approximately quartic, rather than quadratic, would have even $\ddot{a}$ vanishing at $t=0$. To rule out hypothetical NEC-satisfying bounce solutions of this type and close the loophole, consider a Taylor-expansion of the scale factor about $t=0$ :

$$
a(t) \approx a_{*}+c t^{2 n}+\ldots
$$

\footnotetext{
${ }^{1}$ We emphasize here that our discussion is in the context of classical gravity. That is, it is assumed that spacetime curvatures at the bounce are not so high that a full theory of quantum gravity must necessarily be invoked. In other words, the subject is not the resolution of the cosmological singularity by quantum gravity but rather the avoidance of the singularity by means of a classical bounce. This of course is also the context of the majority of papers on bouncing cosmologies in the literature, indeed, whenever the Friedmann equation is used.
} 
Here $a_{*}$ is the scale factor at the bounce and $c$ is a strictly positive constant; the case of interest is $n>1$. Substituting into (1.2) gives

$$
\rho+p \approx \frac{d-1}{8 \pi G_{N}}\left(\frac{k}{a_{*}^{2}}-2 n(2 n-1) c t^{2 n-2}+\ldots\right) .
$$

Evidently, even though $\rho+p$ can be zero at the bounce (for $k=0$ ), $\rho+p$ must be strictly negative for arbitrarily small, nonzero $t$ in order for a bounce solution to exist. The naive conclusion therefore stands: to obtain a bouncing flat or open FRW cosmological solution in classical Einstein gravity, it is necessary that the NEC be violated [4]. ${ }^{2}$

Thus, in Einstein gravity, the possibility of flat or open bouncing cosmologies is intimately connected to the status of the NEC. Let us therefore briefly review the energy conditions. The energy conditions play a vitally important role in general relativity. They are the main local constraints determining which solutions of Einstein's equations are physical; without the energy conditions, every metric would formally constitute an exact solution (at least locally) to Einstein's equations. Moreover, the validity of various energy conditions is the primary assumption in the singularity theorems [5] as well as in no-go theorems that prohibit the traversability of wormholes, the creation of laboratory universes [6], and the building of time machines [7]. Perhaps most importantly, the energy conditions are invoked in the laws of black hole mechanics, where they are used in proving that the area of a black hole event horizon, like entropy, always increases [8]. The most fundamental of the energy conditions is the null energy condition which, in its covariant formulation, states that at every point in spacetime,

$$
T_{\mu \nu} v^{\mu} v^{\nu} \geq 0,
$$

for any light-like vector, $v^{\mu}$. The NEC is the weakest of the energy conditions in that a violation of the NEC automatically implies a violation of the weak, dominant, and strong energy conditions.

Now, the importance of the NEC stems essentially from the null version of Raychaudhuri's equation which governs the focussing of light rays. Raychaudhuri's equation states that a null geodesic congruence with affine parameter $\lambda$, expansion parameter $\theta$, and shear $\sigma_{a b}$ satisfies

$$
\frac{d \theta}{d \lambda}=-\frac{1}{2} \theta^{2}-\sigma_{a b} \sigma^{a b}-R_{\mu \nu} v^{\mu} v^{\nu},
$$

where we have ignored a vorticity term and assumed $d=3$ for simplicity. Thus if

$$
R_{\mu \nu} v^{\mu} v^{\nu} \geq 0
$$

then every term on the right in Raychaudhuri's equation is negative, which is the key requirement in proving a number of gravitational theorems. The problem is that, at least within the framework of general relativity, there is no compelling reason why (1.7), known

\footnotetext{
${ }^{2}$ Of course, for $k=+1$, bounce solutions that satisfy the NEC are easy to find, the simplest example of which is just global de Sitter space with scale factor $a(t)=\frac{1}{\sqrt{b \Lambda}} \cosh (\sqrt{b \Lambda} t)$ where $b=2 /\left(d^{2}-d\right)$. To rule out this case as well, one needs the strong energy condition; however, we will not consider closed universes here.
} 
as the null or Ricci convergence condition, should hold. However, if Einstein's equations are used, then

$$
R_{\mu \nu} v^{\mu} v^{\nu} \geq 0 \Leftrightarrow T_{\mu \nu} v^{\mu} v^{\nu} \geq 0 .
$$

That is, if the null energy condition, (1.5), holds for matter, then the Ricci convergence condition, (1.7), holds for geometry (and vice versa). Attention thus shifts to the matter sector, described by quantum field theory. However, although (1.5) does hold for all familiar forms of matter, it does not appear to have a rigorous derivation, say in the classical limit of quantum field theory. Consequently, the validity of the NEC has been called into question [9, 10]. Indeed, there are known nontrivial NEC-violating effective theories of matter, such as ghost condensates [11] which, although they have peculiar properties, do not have any fatal instabilities at least within the framework of effective field theory. (There could, however, still be issues with negatively unbounded Hamiltonians [12].) Cosmologists have exploited these new theories of matter to obtain bouncing cosmological solutions to Einstein's equations [13-15].

Thus it appears that there is neither an origin in general relativity of (1.7), nor an origin in quantum field theory of (1.5). In fact, this is not altogether surprising. The distinction between matter and gravity is not fundamental: in Kaluza-Klein compactifications, as well as in changes of conformal frame, we see that what appears to be in the matter sector can equally be regarded as being geometry, thereby transforming what we mean by the energy-momentum tensor which in turn transforms the energy conditions [16]. A statement like (1.5) that applies only to matter is unlikely to be fundamental. Moreover, the overall sign of the matter action does not have any meaning in quantum field theory. To obtain conditions like (1.5) or (1.7), we should therefore look to a theory of both matter and gravity, for which the overall sign of the matter action does have meaning since it is now a relative sign, relative to that of the gravitational action.

Indeed, the Ricci convergence condition, (1.7), has recently been derived as a generic classical consequence of string theory [17], a theory which of course does describe both matter and gravity. The purpose of this paper is to point out the implications of that derivation for cosmological bounces. There are two main results. First, an immediate corollary of the derivation of the Ricci convergence condition is that open or flat bouncing cosmological solutions of the classical Einstein equations are not consistent with string theory, quite independent of the existence of well-behaved NEC-violating theories of matter. Second, the regimes in which the derivation is obtained reveal how the no-go theorem might potentially be evaded and so we map out the space of theories in which to look for stringcompatible bouncing cosmologies. We rule out one broad class of such theories, while keeping alive various other approaches in which gravity is modified, or matter is nonminimally coupled, or there are quantum effects, or there are orientifolds present. Let us therefore briefly review the derivation of the Ricci convergence condition.

\section{Derivation of the Ricci convergence condition}

Worldsheet string theory is described by a two-dimensional nonlinear sigma model in which $D$ scalar fields, $X^{\mu}(\sigma, \tau)$ — we focus here on bosonic string theory — are minimally coupled 
to two-dimensional Einstein gravity on the worldsheet. The Polyakov action for a string propagating in flat space is

$$
S_{P}\left[X^{\mu}, h_{a b}\right]=-\frac{1}{4 \pi \alpha^{\prime}} \int d^{2} \sigma \sqrt{-h}\left[\alpha^{\prime} c R_{h}+h^{a b} \partial_{a} X^{\mu} \partial_{b} X^{\nu} \eta_{\mu \nu}(X)\right],
$$

where $R_{h}$ is the worldsheet Ricci scalar, and $c$ is an arbitrary constant. The equation of motion for the worldsheet metric, $h_{a b}$, is just Einstein's equation in two dimensions:

$$
0=T_{a b}^{\mathrm{ws}} .
$$

Here the left-hand side is zero because the Einstein tensor vanishes identically in two dimensions, and $T_{a b}^{\mathrm{ws}}$ is the energy-momentum tensor on the worldsheet:

$$
T_{a b}^{\mathrm{ws}}=\frac{1}{2 \pi \alpha^{\prime}}\left(\partial_{a} X^{\mu} \partial_{b} X^{\nu} \eta_{\mu \nu}-\frac{1}{2} h_{a b}(\partial X)^{2}\right) .
$$

Switching to light-cone coordinates on the worldsheet,

$$
\sigma^{ \pm} \equiv \tau \pm \sigma
$$

we see that the diagonal components of the worldsheet metric vanish:

$$
h_{a b}=\left(\begin{array}{cc}
0 & -\frac{1}{2} \\
-\frac{1}{2} & 0
\end{array}\right) \text {. }
$$

The two-dimensional Einstein equation (2.2) then reads

$$
0=\partial_{+} X^{\mu} \partial_{+} X^{\nu} \eta_{\mu \nu}
$$

with a similar equation holding when + is replaced by -. These are the Virasoro constraints. Defining a vector field $v_{+}^{\mu}=\partial_{+} X^{\mu}(\sigma, \tau)$, we find that

$$
\eta_{\mu \nu} v_{+}^{\mu} v_{+}^{\nu}=0
$$

which is to say that $v_{+}^{\mu}$ is a null vector field. The key point here is that worldsheet string theory naturally singles out spacetime null vectors.

Next we will see how these lead to the Ricci convergence condition when the string propagates in an arbitrary curved spacetime. The Polyakov action is now

$$
S_{P}\left[X^{\mu}, h_{a b}\right]=-\frac{1}{4 \pi \alpha^{\prime}} \int d^{2} \sigma \sqrt{-h}\left[\alpha^{\prime} \Phi(X) R_{h}+h^{a b} \partial_{a} X^{\mu} \partial_{b} X^{\nu} g_{\mu \nu}(X)\right] .
$$

We have replaced the Minkowski metric $\eta_{\mu \nu}$ by the general spacetime metric $g_{\mu \nu}(X)$. Consistent with worldsheet diffeomorphism-invariance, we have also allowed there to be a scalar field, $\Phi(X(\tau, \sigma))$, which is the dilaton field; we neglect the anti-symmetric Kalb-Ramond field, $B_{\mu \nu}$, for simplicity.

We now perform a background field expansion $X^{\mu}(\tau, \sigma)=X_{0}^{\mu}(\tau, \sigma)+Y^{\mu}(\tau, \sigma)$ where $X_{0}^{\mu}(\tau, \sigma)$ is some solution of the classical equation of motion. Then, for every value of 
$(\tau, \sigma)$, we can use standard field redefinitions $[18,19]$ to expand the metric in Riemann normal coordinates about the spacetime point $X_{0}^{\mu}(\tau, \sigma)$ :

$$
g_{\mu \nu}(X)=\eta_{\mu \nu}-\frac{1}{3} R_{\mu \alpha \nu \beta}\left(X_{0}\right) Y^{\alpha} Y^{\beta}-\ldots
$$

Contracted with $\partial_{a} X^{\mu} \partial^{a} X^{\nu}$, the second and higher terms introduce quartic and higher terms in the Lagrangian; spacetime curvature turns (2.8) into an interacting theory. The resultant divergences can be cancelled by adding suitable counter-terms to the original Lagrangian. Integrating out $Y$, the one-loop effective action is $[18,19]$

$$
S\left[X_{0}^{\mu}, h_{a b}\right]=-\frac{1}{4 \pi \alpha^{\prime}} \int d^{2} \sigma \sqrt{-h}\left[\alpha^{\prime} C_{\epsilon} \Phi R_{h}+h^{a b} \partial_{a} X_{0}^{\mu} \partial_{b} X_{0}^{\nu}\left(\eta_{\mu \nu}+C_{\epsilon} \alpha^{\prime} R_{\mu \nu}\right)\right] .
$$

Here $C_{\epsilon}$ is the divergent coefficient of the counter-terms.

In light-cone coordinates, the Virasoro constraint now reads

$$
0=\partial_{ \pm} X_{0}^{\mu} \partial_{ \pm} X_{0}^{\nu}\left(\eta_{\mu \nu}+C_{\epsilon} \alpha^{\prime} R_{\mu \nu}+2 C_{\epsilon} \alpha^{\prime} \nabla_{\mu} \nabla_{\nu} \Phi\right)
$$

Consider then an arbitrary null vector $v^{\mu}$ in the tangent plane of some arbitrary point in an arbitrary spacetime. Let there be a test string passing through the given point with $\partial_{+} X^{\mu}$ equal to $v^{\mu}$ at the point. Note that at every point $X_{0}^{\mu}(\tau, \sigma)$ the spacetime metric $g_{\mu \nu}(X)$ is just $\eta_{\mu \nu}$; for a string passing through such a point, $\partial_{+} X^{\mu}$ is therefore null with respect to both $\eta_{\mu \nu}$ and $g_{\mu \nu}(X)$. Defining $v_{+}^{\mu}=\partial_{+} X^{\mu}$ as before, we find (2.7) at leading order in $\alpha^{\prime}$. At the next order, we have

$$
v_{+}^{\mu} v_{+}^{\nu}\left(R_{\mu \nu}+2 \nabla_{\mu} \nabla_{\nu} \Phi\right)=0
$$

This is tantalizingly close to our form of the Ricci convergence condition, (1.7), except for two differences: it is an equality, rather than an inequality, and there is an additional, unwanted term involving the dilaton.

However, now we recall that the metric that appears in the worldsheet action is the string-frame metric. We can transform to Einstein frame by defining:

$$
g_{\mu \nu}=e^{\frac{4 \Phi}{D-2}} g_{\mu \nu}^{E} .
$$

Then we find that

$$
R_{\mu \nu}^{E} v_{+}^{\mu} v_{+}^{\nu}=+\frac{4}{D-2}\left(v_{+}^{\mu} \nabla_{\mu}^{E} \Phi\right)^{2} .
$$

The right-hand side is manifestly non-negative. Hence we have

$$
R_{\mu \nu}^{E} v_{+}^{\mu} v_{+}^{\nu} \geq 0
$$

This establishes the null or Ricci convergence condition, which is equivalent to the null energy condition when Einstein's equations hold.

In summary, the spacetime interpretation of the Virasoro constraint on the worldsheet is precisely the Ricci convergence condition [17]. This result was obtained in the critical number of spacetime dimensions ( $D=26$ for a bosonic string) because the worldsheet oneloop effective action we used was for a string moving in the critical number of dimensions. 
But it is easy to show that the result holds in lower dimensions too. For a string propagating in a non-critical number of dimensions, there is an extra term in the worldsheet energy momentum-tensor. However, the extra term is proportional to $h_{a b}$ and therefore does not contribute to $(2.11)$.

\section{Implications for bouncing cosmologies}

We have seen that the Ricci convergence condition is rather essential to string theory. It is connected to a principle - worldsheet gravity - rather than being an unexplained, accidental fact the way the null energy condition is for most theories, including supergravity. Nor does it have anything to do with supersymmetry: we were able to derive it in bosonic string theory. Hence it is a very general requirement which we cannot lightly discard. Let us then apply $R_{\mu \nu} v^{\mu} v^{\nu} \geq 0$ directly to a $k \neq 1$ FRW cosmology. In standard RobertsonWalker coordinates, we have

$$
\left(v^{t}\right)^{2}=a^{2}(t) \vec{v}^{2},
$$

where $v^{a}=\left(v^{t}, \vec{v}\right)$ is any null vector. Then we find that

$$
R_{\mu \nu} v^{\mu} v^{\nu}=\vec{v}^{2} a^{2}(t)(d-1)\left(-\dot{H}+\frac{k}{a^{2}}\right) .
$$

Imposing (1.7), we arrive at our first main result: (1.7) directly establishes that, for $k \neq 1$, $\dot{H}$ cannot be positive, thereby precluding a bounce solution.

We would like to emphasize the novelty of this approach: the usual way to rule out bounces would be by invoking the NEC, a property of matter, and then using the Friedmann equation. However, as we have seen, the NEC on its own has not been established from QFT and, moreover, there appear to be plausibly well-behaved theories of matter that violate the NEC. Instead, we have shown above that string theory gives a constraint directly at the level of geometry; a closed string simply cannot propagate consistently in such bouncing universes because the Virasoro constraints would be violated on the worldsheet. In other words, regardless of the possible existence of NEC-violating matter, closed strings are not compatible with the metric corresponding to an open or flat bouncing FRW universe. In summary, consistency with string theory rules out open or flat bouncing FRW solutions to leading order in $\alpha^{\prime}$, independently of (so far unprovable) assumptions about matter satisfying the null energy condition. The necessity of the Ricci convergence condition in string theory shuts the door on the simplest kinds of bounce cosmologies, in which some exotic NEC-violating matter field is minimally coupled to Einstein gravity. Such solutions are not consistent with low-energy string theory.

But the derivation also reveals the limitations of the claim. This then brings us to the second point of this paper, which is to identify the regimes of validity of the calculation in order to classify the remaining options for finding potential string-compatible bounces. In that context, note in particular that we derived the Ricci convergence condition while working to the lowest order in alpha prime and to the lowest genus in the string genus expansion. In field theory language, these two regimes correspond to working at leading order in the curvature expansion and at tree-level in the loop expansion. Consider first 
the effect of radiative corrections. It is tempting to write down the semi-classical Einstein equations:

$$
G_{\mu \nu}=8 \pi G_{N}\left\langle T_{\mu \nu}\right\rangle-\Lambda g_{\mu \nu},
$$

where $\left\langle T_{\mu \nu}\right\rangle$ is the renormalized stress tensor of matter calculated in some state in a given background. There is, to our knowledge, no rigorous derivation of such an equation starting from a theory that treats both gravity and matter quantum-mechanically. If such an equation were nevertheless to exist, it would offer the possibility of bounce solutions. The left-hand side would still lead to the left-hand sides of (1.1) and (1.2), but the right-hand side would presumably no longer obey the null energy condition even for matter that obeys it classically. Indeed, even for ordinary fields, Casimir energy can cause $\left\langle T_{\mu \nu}\right\rangle$ to violate the NEC. However, although (3.3) appears plausibly true as a semi-classical approximation, it is not obvious that it is consistent with string theory either. This is because (3.3) treats gravity classically while treating matter quantum-mechanically. Yet in closed string theory, the graviton is just another mode of the string, so such an unequal treatment of gravity and matter is in tension, at the minimum, with the spirit of string theory.

The other, perhaps more promising, direction is to modify the geometric part of Einstein's equations. There are several ways this can be used to find bounces, such as nonminimal coupling to matter [20] including kinetic-gravity braiding [21], massive gravity [22], or the inclusion of torsion [23]. The most obvious extension is to add higher-curvature terms to the gravitational equations [24]. The presence of these terms is expected not only because Einstein gravity is not renormalizable, but also because the low-energy effective action of string theory naturally contains such terms at higher order in the alpha-prime expansion. When higher-curvature terms are included, two things happen. First, the string-frame Virasoro constraint condition gets modified:

$$
v_{+}^{\mu} v_{+}^{\nu}\left(R_{\mu \nu}+2 \nabla_{\mu} \nabla_{\nu} \Phi+\text { higher-curvature }\right)=0 .
$$

Upon transforming to Einstein frame, the Virasoro constraint on the worldsheet no longer translates to the Ricci convergence condition in spacetime, which is the condition it is necessary to relax if flat or open FRW cosmologies are to have bounces. Second, since the geometric part of Einstein's equations is modified, (1.8) no longer holds. As a concrete example of how the physics changes in higher-curvature gravity, consider heterotic string theory. To sub-leading order in $\alpha^{\prime}$, the low-energy effective action contains the Gauss-Bonnet term [25]. It is known that, for Gauss-Bonnet gravity, the scalar obtained under dimensional reduction is a galileon [26]. Thus, we have a potential example of a lower-dimensional NEC-violating theory of matter that comes from string theory. This would not contradict [17] because the resulting galileons would not be coupled to Einstein gravity but to Einstein-Gauss-Bonnet gravity. But note that any new terms in the gravitational equations that are not proportional to the metric break the equivalence between (1.7) and (1.5). NEC violation then becomes neither necessary nor sufficient to having a cosmological bounce solution to the gravitational equations of motion. It would be interesting to see whether galileons coupled to Einstein-Gauss-Bonnet gravity still have bounce solutions. In any case, higher-curvature gravity appears to offer the best chance to find string-compatible bouncing cosmologies with or without NEC-violation. 
Finally, there could be implicit assumptions in our proof. (Of course one such assumption is that the correct theory of matter and gravity is string theory.) For example, in (2.9) we assumed that the metric can be expanded about the spacetime point $X_{0}^{\mu}(\tau, \sigma)$ in Riemann normal coordinates, which is the case if there is an open coordinate patch around the point. However, as noted in [17], that is not true if the point lies on a boundary of spacetime. Thus potential NEC violations may be allowed if spacetime has a boundary. It is noteworthy in this context that string theory contains orientifolds, extended objects with negative tension which violate the NEC and which are non-dynamical branes that typically live on a boundary of spacetime. These may have interesting applications to cosmology including the search for viable bounce solutions [27, 28].

In conclusion, we have shown in this paper that open or flat bouncing FRW cosmologies are inconsistent with string theory. The lack of a first-principles derivation of the null energy condition from quantum field theory, combined with the existence of NEC-violating stable effective theories of matter, have led to many scenarios in which such matter is used to support a cosmological bounce, when it is coupled by hand to Einstein gravity. Here we have shown, as a corollary of our earlier work, that the null or Ricci convergence condition which follows from string theory rules out such cosmologies, irrespective of the status of NEC-violating matter. This is a result that could not have been derived from either general relativity or quantum field theory, since the sign of (1.5) or (1.7) is only meaningful in a theory containing both matter and gravity. But we also showed that there are potentially ways to bypass this no-go theorem by venturing beyond Einstein gravity. We have therefore ruled out certain classes of bouncing cosmologies while leaving the door open for bounce solutions of extensions of Einstein gravity.

\section{Acknowledgments}

M.P. is supported in part by DOE grant DE-FG02-09ER41624.

Open Access. This article is distributed under the terms of the Creative Commons Attribution License (CC-BY 4.0), which permits any use, distribution and reproduction in any medium, provided the original author(s) and source are credited.

\section{References}

[1] R.C. Tolman, On the Theoretical Requirements for a Periodic Behaviour of the Universe, Phys. Rev. 38 (1931) 1758.

[2] M. Novello and S.E.P. Bergliaffa, Bouncing Cosmologies, Phys. Rept. 463 (2008) 127 [arXiv:0802.1634] [INSPIRE].

[3] D. Battefeld and P. Peter, A Critical Review of Classical Bouncing Cosmologies, Phys. Rept. 571 (2015) 1 [arXiv: 1406.2790] [INSPIRE].

[4] C. Molina-París and M. Visser, Minimal conditions for the creation of a Friedman-Robertson-Walker universe from a 'bounce', Phys. Lett. B 455 (1999) 90 [gr-qc/9810023] [INSPIRE]. 
[5] R. Penrose, Gravitational collapse and space-time singularities, Phys. Rev. Lett. 14 (1965) 57 [INSPIRE].

[6] E. Farhi and A.H. Guth, An Obstacle to Creating a Universe in the Laboratory, Phys. Lett. B 183 (1987) 149 [INSPIRE].

[7] S.W. Hawking, The Chronology protection conjecture, Phys. Rev. D 46 (1992) 603 [InSPIRE].

[8] J.M. Bardeen, B. Carter and S.W. Hawking, The Four laws of black hole mechanics, Commun. Math. Phys. 31 (1973) 161 [INSPIRE].

[9] C. Barcelo and M. Visser, Twilight for the energy conditions?, Int. J. Mod. Phys. D 11 (2002) 1553 [gr-qc/0205066] [INSPIRE].

[10] V.A. Rubakov, The Null Energy Condition and its violation, Phys. Usp. 57 (2014) 128 [arXiv: 1401.4024] [INSPIRE].

[11] N. Arkani-Hamed, H.-C. Cheng, M.A. Luty and S. Mukohyama, Ghost condensation and a consistent infrared modification of gravity, JHEP 05 (2004) 074 [hep-th/0312099] [INSPIRE].

[12] I. Sawicki and A. Vikman, Hidden Negative Energies in Strongly Accelerated Universes, Phys. Rev. D 87 (2013) 067301 [arXiv: 1209.2961] [INSPIRE].

[13] Y.-F. Cai, T. Qiu, Y.-S. Piao, M. Li and X. Zhang, Bouncing universe with quintom matter, JHEP 10 (2007) 071 [arXiv:0704.1090] [INSPIRE].

[14] T. Qiu, J. Evslin, Y.-F. Cai, M. Li and X. Zhang, Bouncing Galileon Cosmologies, JCAP 10 (2011) 036 [arXiv:1108.0593] [INSPIRE].

[15] C. Lin, R.H. Brandenberger and L. Perreault Levasseur, A Matter Bounce By Means of Ghost Condensation, JCAP 04 (2011) 019 [arXiv: 1007.2654] [InSPIRE].

[16] S. Chatterjee, D.A. Easson and M. Parikh, Energy conditions in the Jordan frame, Class. Quant. Grav. 30 (2013) 235031 [arXiv:1212.6430] [INSPIRE].

[17] M. Parikh and J.P. van der Schaar, Derivation of the Null Energy Condition, Phys. Rev. D 91 (2015) 084002 [arXiv:1406.5163] [InSPIRE].

[18] C.G. Callan Jr. and L. Thorlacius, Sigma Models and String Theory, in Particles, Strings and Supernovae: Proceedings of the Theoretical Advanced Study Institute in Elementary Particle Physics (TASI 88), edited by A. Jevicki and C.I. Tan, World Scientific, Teaneck, N.J. (1989).

[19] M.B. Green, J.H. Schwarz and E. Witten, Superstring Theory: Introduction, Cambridge, London U.K. (1987).

[20] M. Gasperini and G. Veneziano, The Pre-big bang scenario in string cosmology, Phys. Rept. 373 (2003) 1 [hep-th/0207130] [INSPIRE].

[21] D.A. Easson, I. Sawicki and A. Vikman, G-Bounce, JCAP 11 (2011) 021 [arXiv:1109.1047] [INSPIRE].

[22] D. Langlois and A. Naruko, Bouncing cosmologies in massive gravity on de Sitter, Class. Quant. Grav. 30 (2013) 205012 [arXiv: 1305.6346] [INSPIRE].

[23] Y.-F. Cai, S.-H. Chen, J.B. Dent, S. Dutta and E.N. Saridakis, Matter Bounce Cosmology with the $f(T)$ Gravity, Class. Quant. Grav. 28 (2011) 215011 [arXiv:1104.4349] [InSPIRE].

[24] R.H. Brandenberger, V.F. Mukhanov and A. Sornborger, A Cosmological theory without singularities, Phys. Rev. D 48 (1993) 1629 [gr-qc/9303001] [InSPIRE]. 
[25] K.A. Meissner, Symmetries of higher order string gravity actions, Phys. Lett. B 392 (1997) 298 [hep-th/9610131] [INSPIRE].

[26] K. Van Acoleyen and J. Van Doorsselaere, Galileons from Lovelock actions, Phys. Rev. D 83 (2011) 084025 [arXiv: 1102.0487] [INSPIRE].

[27] L. Cornalba, M.S. Costa and C. Kounnas, A Resolution of the cosmological singularity with orientifolds, Nucl. Phys. B 637 (2002) 378 [hep-th/0204261] [InSPIRE].

[28] L. Cornalba and M.S. Costa, Time dependent orbifolds and string cosmology, Fortsch. Phys. $\mathbf{5 2}$ (2004) 145 [hep-th/0310099] [INSPIRE]. 